\title{
Management of tricuspid regurgitation in congenital heart disease: Is survival better with valve repair?
}

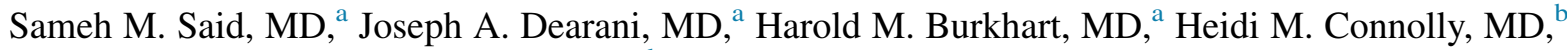 \\ Ben Eidem, MD, ${ }^{\mathrm{c}}$ Paul E. Stensrud, MD, ${ }^{\mathrm{d}}$ and Hartzell V. Schaff, $\mathrm{MD}^{\mathrm{a}}$
}

\begin{abstract}
Objective: Tricuspid valve (TV) regurgitation in congenital heart disease includes a heterogeneous group of lesions, and few series have documented the outcomes.

Methods: We reviewed the records of 553 patients with congenital heart disease who had undergone TV surgery for tricuspid regurgitation from January 1993 to December 2010. Patients with Ebstein malformation were excluded. Their mean age was $32 \pm 21$ years, and 300 were female $(54 \%)$. The most common diagnoses were conotruncal anomaly in 216 patients $(39 \%)$, previous ventricular septal defect closure in $83(15 \%)$, atrioventricular septal defect in $77(14 \%)$, and pulmonary atresia with an intact ventricular septum in 11 $(2 \%)$. Preoperative right-sided heart failure was present in 124 patients $(22 \%)$, and 55 patients $(10 \%)$ had pulmonary hypertension.
\end{abstract}

Results: TV repair was performed in $442(80 \%)$ and TV replacement in $111(20 \%)$ patients. Repeat sternotomy was performed in 415 patients (75\%). Previous TV repair was present in 44 patients (8\%); of these, $17(38.6 \%)$ underwent repeat TV repair. The overall early mortality was 3.1\% (17 patients) and was $2.5 \%$ for TV repair and $5.4 \%$ for TV replacement $(P=.001)$. The mean follow-up period was $4.5 \pm 4.1$ years (maximum, 18$)$. The overall survival at 1,5 , and 10 years was $97 \%, 93 \%$, and $85 \%$, respectively. Survival was better for patients with repair than with replacement. TV repair was an independent predictor of better survival $(P=.001)$.

Conclusions: Important tricuspid regurgitation can occur with a variety of congenital diagnoses. Early mortality is low and late survival is superior with tricuspid repair than with valve replacement. Surgical treatment of tricuspid regurgitation in congenital heart disease should be performed before the onset of heart failure. (J Thorac Cardiovasc Surg 2014;147:412-9)

Congenital (non-Ebstein) tricuspid regurgitation (TR) is more common than previously thought. It occurs with a variety of congenital diagnoses and requires a unique management strategy. ${ }^{1}$ The etiologies can include congenital tricuspid valve (TV) dysplasia, such as in cases of pulmonary atresia with an intact ventricular septum, and in association with other congenital heart defects, such as atrioventricular septal defect (AVSD) or pulmonary stenosis with ventricular septal defect (VSD), or as a result of annular dilatation secondary to right ventricular (RV) volume overload (eg, atrial septal defect, anomalous pulmonary venous connection, or pulmonary regurgitation). Iatrogenic TR in the congenital population can be encountered after previous VSD closure or secondary to pacemaker or

From the Divisions of Cardiovascular Surgery, ${ }^{\mathrm{a}}$ Cardiovascular Diseases, ${ }^{\mathrm{b}}$ Pediatric Cardiology, ${ }^{\mathrm{c}}$ and Cardiac Anesthesia, ${ }^{\mathrm{d}}$ Mayo Clinic, Rochester, Minn.

Disclosures: Authors have nothing to disclose with regard to commercial support.

Read at the 93rd Annual Meeting of The American Association for Thoracic Surgery, Minneapolis, Minnesota, May 4-8, 2013.

Received for publication May 6, 2013; revisions received July 18, 2013; accepted for publication Aug 11, 2013; available ahead of print Sept 30, 2013.

Address for reprints: Joseph A. Dearani, MD, Division of Cardiovascular Surgery, Mayo Clinic, 200 First St SW, Rochester, MN 55905 (E-mail: dearani.joseph@ mayo.edu).

$0022-5223 / \$ 36.00$

Copyright (c) 2014 by The American Association for Thoracic Surgery

http://dx.doi.org/10.1016/j.jtcvs.2013.08.034 implantable cardiac defibrillator (ICD) placement (Table 1). Few data have been published that provide the outcomes of TV surgery in the congenital population. We reviewed our experience with TV surgery in this heterogeneous group of patients.

\section{METHODS}

The Mayo Foundation institutional review board approved the present study, and all patients provided informed consent. From January 1993 through December 2010, 571 patients with congenital heart disease underwent surgery for TV regurgitation. Eighteen patients were excluded because of an inability to obtain informed consent. The records of the remaining 553 patients were reviewed. Patients with Ebstein malformation (EM), a functional single ventricle, and congenitally corrected transposition of the great arteries were excluded. The mean age was $32 \pm 21$ years, and 300 patients were female $(54 \%)$.

The most common congenital diagnoses were conotruncal abnormalities in 216 patients (39\%), previous VSD closure in 83 patients $(15 \%)$, AVSD in 77 patients (14\%), pulmonary atresia (PA) with an intact septum in 11, and TR secondary to pacemaker or ICD placement was present in 67 patients $(12 \%)$. The remaining 99 patients $(18 \%)$ had TR secondary to annular dilatation from RV volume overload, such as in a partial anomalous pulmonary venous connection, septal defects, and coronary artery fistulas.

Preoperative congestive heart failure was present in 124 patients (22\%), 55 patients $(10 \%)$ had significant pulmonary hypertension (more than one half, systemic), and 37 (7\%) had a history of endocarditis (Table 2). One or more previous sternotomies $(\leq 8)$ was present in 415 patients $(75 \%)$. Forty-four patients $(8 \%)$ had undergone previous TV repair, and repeat repair was achieved in $17(39 \%)$. 


$$
\begin{aligned}
& \text { Abbreviations and Acronyms } \\
& \begin{aligned}
\text { AVSD } & =\text { atrioventricular septal defect } \\
\text { EM } & =\text { Ebstein malformation } \\
\text { ICD } & =\text { implantable cardiac defibrillator } \\
\text { TR } & =\text { tricuspid regurgitation } \\
\text { TV } & =\text { tricuspid valve } \\
\text { VSD } & =\text { ventricular septal defect }
\end{aligned}
\end{aligned}
$$

The main indication for surgery in all cases was right-sided heart disease. The indication for TV intervention was the presence of severe TR with symptoms that included fatigue, decreased stamina, reduced exercise tolerance, or right-sided heart failure. Other indications included progressive RV enlargement with or without RV dysfunction or the onset of atrial arrhythmia. Concomitant right-sided procedures included pulmonary valve replacement (PVR) in 207 (37\%) and RV-PA conduit replacement in $37(7 \%)$. Concomitant left-sided procedures for lesions that were moderate to severe that were addressed at surgery included mitral valve repair in $80(14 \%)$, mitral valve replacement in $44(8 \%)$, and aortic valve replacement in $25(5 \%)$.

Our goal was to repair the TV whenever possible. Annular dilatation was managed primarily by annuloplasty-eccentric (inferior annulus), pursestring DeVega from the anteroseptal to inferoseptal commissure, or a flexible c-shaped band from the anteroseptal commissure to the coronary sinus. When structural abnormalities were present in the leaflets or subvalvular apparatus, we applied some of the principles of the cone reconstruction for EM. ${ }^{2}$ This included mobilization of the leaflet tissue and subvalvular apparatus when needed. Other modifications for TV repair in EM included (1) approximation of the head of the mobilized "baseintact" RV free wall papillary muscle to a corresponding head of a papillary muscle arising from the septum supporting the septal leaflet (modified Sebening stitch); (2) distal surgical fenestrations to create autologous neochordae when a linear attachment or tethering of the leading edge was present in cases of TV dysplasia; (3) placement of Gore-Tex (W. L. Gore \& Associates, Flagstaff, Ariz) artificial chordae; and (4) tricuspid leaflet augmentation with a CorMatrix membrane (Cook Biotech, West Lafayette, Ind) or autologous pericardium to increase the leaflet height.

A c-shaped flexible annuloplasty band (anteroseptal commissure clockwise to the coronary sinus) was preferred when valve stenosis was not a concern. The use of a ring was possible in many younger patients without creating tricuspid stenosis, because the annulus was often quite dilated; thus, the normal adult annulus size of 20 to $22 \mathrm{~mm}$ could be achieved in most children older than about 10 years. When the annulus size and potential stenosis were a concern, a limited eccentric band from the anteroinferior commissure to the coronary sinus was used, because this is the area of the annulus most susceptible to dilatation and the area under the greatest tension.

TV replacement was performed in 111 patients $(20 \%)$ when repair was not feasible, particularly in adults. Our preference has been a porcine bioprosthesis because of its relative good durability in the tricuspid position and the lack of a need for chronic anticoagulation. In the present study, 92 patients $(83 \%)$ underwent bioprosthetic TV replacement, and 19 patients $(17 \%)$ received a mechanical prosthesis. Mechanical prostheses were used when the RV function was good and the patient had another need for chronic warfarin anticoagulation (eg, left-sided mechanical prosthesis). The technique of prosthesis placement included retaining the tricuspid leaflet and subvalvular apparatus whenever possible. Prosthesis suture placement was in the native leaflet tissue to minimize injury to the conduction tissue and right coronary artery. When the leaflet tissue was thickened and dysplastic and required resection, a rim of leaflet tissue adjacent to the annulus was preserved to facilitate valve suture placement. In contrast to TV replacement for EM, the coronary sinus was always left draining into the right atrium, because the prosthesis was anchored in the atrioventricular groove into native tricuspid leaflet tissue. In the setting of TV leaflet regurgitation secondary to pacemaker or ICD leads, TV replacement was performed as described, and the ventricular lead was preserved (when the thresholds were excellent) and positioned lateral to the sewing ring of the prosthesis. This technique involved folding the leaflet and annulus tissue in the area of the lead (the inferoseptal commissure was the most common) and then placing valve sutures. This enabled the lead to move freely outside the sewing ring of the valve. When pacemaker lead malfunction occurred, the ventricular lead was removed, and an epicardial system was placed at TV surgery.

The medical records were reviewed to obtain the demographic characteristics, associated medical conditions, details of the operations, and results of the hemodynamic and echocardiographic testing.

\section{Statistical Analysis}

The descriptive statistics for categorical variables are reported as the frequency and percentage. Continuous variables are reported as the mean \pm standard deviation or median and range, as appropriate. Continuous variables were compared using the 2-sample $t$ test or the Wilcoxon rank sum test, as appropriate. The Kaplan-Meier method was used to draw survival curves and calculate survival statistics at $1,5,10$, and 15 years. Univariate and multivariate Cox regression models were used to identify the factors associated with overall death. All the tests were 2-sided. $P<.05$ was considered statistically significant. Statistical Analysis Systems, version 9.13, software (SAS Institute, Cary, NC) was used for statistical analysis.

\section{RESULTS}

Of the 553 patients, TV repair was performed in $442(80 \%)$ and valve replacement in $111(20 \%)$. The overall early mortality was $3.1 \%$ (17 patients). Early mortality was greater after TV replacement (6 patients, 5.4\%) than after TV repair (11 patients, $2.5 \% ; P=.001)$. In the 448 patients who had undergone $\leq 3$ sternotomies, $82 \%$ underwent TV repair. Of the 105 patients who had undergone $\geq 4$ sternotomies, $35 \%$ required TV replacement. Delayed sternal closure was performed in 35 patients $(6 \%)$. No risk factors for early mortality were identified. Early postoperative morbidity included mechanical ventilation $>5$ days $(n=27 ; 5 \%)$, permanent pacemaker placement $(\mathrm{n}=23 ; 4 \%)$, renal failure $(\mathrm{n}=20$; $4 \%)$, stroke $(\mathrm{n}=8 ; 1 \%)$, and sternal infection $(\mathrm{n}=6 ; 1 \%)$.

The mean follow-up period was $4.5 \pm 4.1$ years (maximum, 18). The univariate and multivariate risk factors for late survival are listed in Tables 3 and 4, respectively. Overall survival at 1,5 , and 10 years was $97 \%, 93 \%$, and $85 \%$, respectively (Figure $1, A$ ). Survival was significantly better for the patients with TV repair than for those with TV replacement $(P<.0001$; Figure $1, B)$. The 1-, 5-, and 10 -year survival for TV repair was $98 \%, 96 \%$, and $89 \%$ and for replacement was $91 \%, 81 \%$, and $72 \%$, respectively. No significant difference was found in survival between those who had received a bioprosthetic versus mechanical valve $(P=.77$; Figure 2$)$.

\section{DISCUSSION}

Congenital (non-EM) TV regurgitation includes a wide variety of structural lesions that can include TV dysplasia, 
TABLE 1. Etiologies of congenital (non-Ebstein) tricuspid regurgitation

Primary TV abnormalities (TV dysplasia)

TV dysplasia in pulmonary atresia with intact septum

Congenitally unguarded TV

Uhl's anomaly

Secondary TV abnormalities

Atrioventricular septal defect

Right ventricular outflow tract obstructive lesions

Tetralogy of Fallot

Pulmonary atresia with ventricular septal defect

Pulmonary stenosis with ventricular septal defect

Tricuspid regurgitation with ventricular septal defect

Gerbode defect

Tricuspid annular dilatation (volume overload)

Coronary artery fistulas

Atrial/ventricular septal defect

Partial anomalous pulmonary venous connection

Iatrogenic TR

Previous ventricular septal defect closure (with or without leaflet detachment)

Pacemaker/cardioverter defibrillator lead

$T V$, Tricuspid valve; $T R$, tricuspid regurgitation.

AVSD, or RV outflow tract obstructive lesions (PA/stenosis with an intact ventricular septum), leaflet distortion secondary to an unclosed VSD, or chordal disruption secondary to previous VSD closure. TR due to annular dilatation can also be found with congenital abnormalities associated with RV volume overload, such as in partial anomalous pulmonary venous connection, atrial septal defect, pulmonary regurgitation, and coronary artery fistulas or RV outflow tract obstruction resulting in an elevated RV pressure. ${ }^{3}$ Finally, with the increasing need for either permanent pacing or ICD placement in the congenital population during late follow-up, severe TR can occur; this has often been referred to as "lead-induced" TR.

Increasing evidence from acquired heart failure studies has shown that severe TR results in reduced late survival and is associated with the onset of symptoms, atrial fibrillation, and heart failure. ${ }^{4,5}$ Consequently, an increased awareness of the potential detrimental effect of significant TR in the congenital heart population is essential for clinicians caring for these patients.

In our practice, the indications for TV surgery include symptoms (eg, fatigue, shortness of breath, edema, heart failure; failure to thrive in an infant or child), decreased exercise tolerance, a structural valve abnormality (eg, ruptured chordae, leaflet defect), progressive RV dilatation, and the onset or progression of atrial tachyarrhythmia. Surgery should be advised before the onset of significant RV dysfunction. Finally, we would recommend earlier surgery if the valve anatomy is favorable for a good TV repair. In the setting of severe pulmonary regurgitation, we have used the development or progression of TR related to annular dilatation as an indication for PVR, in addition to increased RV dimensions. In addition to PVR, we repair the TV when TR is severe or when it is moderate with severe annular dilatation on the preoperative echocardiogram. Importantly, the decision to repair the TV should be made preoperatively, because the conditions of anesthesia generally reduce the TR by at least 1 grade.

There are 2 categories of TV dysplasia, 1 with displacement, which is associated with EM, and 1 without displacement. TV dysplasia includes leaflet and/or subvalvular apparatus thickening or tethering. ${ }^{6}$ Similarly, significant

TABLE 2. Baseline characteristics of study population

\begin{tabular}{|c|c|c|c|c|}
\hline Characteristic & All patients $(n=553)$ & $\operatorname{TVR}(\mathbf{n}=111)$ & TV repair $(n=442)$ & $P$ value \\
\hline Age (y) & $32 \pm 21$ & $40 \pm 20$ & $29 \pm 20$ & $<.001$ \\
\hline Male gender & $253(46)$ & $56(50)$ & $197(45)$ & .27 \\
\hline Era of surgery & & & & .18 \\
\hline 1993-1995 & $29(5)$ & $3(3)$ & $26(6)$ & \\
\hline 1995-2010 & $524(95)$ & $108(97)$ & $416(94)$ & \\
\hline NYHA class & & & & .005 \\
\hline II & $429(78)$ & $75(68)$ & $354(80)$ & \\
\hline III or IV & $124(22)$ & $36(32)$ & $88(20)$ & \\
\hline History of endocarditis & $37(7)$ & $8(7)$ & $29(7)$ & .81 \\
\hline Resternotomy & $415(75)$ & $96(87)$ & $319(71)$ & $<.001$ \\
\hline Previous palliation & $121(22)$ & $30(27)$ & $91(21)$ & .14 \\
\hline \multicolumn{5}{|l|}{ Diagnosis } \\
\hline Conotruncal anomalies & $216(39)$ & $28(25)$ & $188(43)$ & .12 \\
\hline AVSD & 77 (14) & $11(10)$ & $66(15)$ & .19 \\
\hline $\mathrm{PA} / \mathrm{IVS}$ & $11(2)$ & $5(5)$ & $6(1)$ & .03 \\
\hline Annular dilatation & $99(18)$ & $43(39)$ & $56(13)$ & .06 \\
\hline Previous VSD repair & $83(15)$ & $32(29)$ & $51(12)$ & .95 \\
\hline Lead-induced & $67(12)$ & $36(32)$ & $31(7)$ & $<.001$ \\
\hline
\end{tabular}

Data presented as mean \pm standard deviation or $\mathrm{n}(\%)$. TVR, Tricuspid valve replacement; $T V$, tricuspid valve; $N Y H A$, New York Heart Association; $A V S D$, atrioventricular septal defect; $P A / I V S$, pulmonary atresia with intact interventricular septum; $V S D$, ventricular septal defect. 
TABLE 3. Univariate hazard ratios for late death for all patients $(\mathbf{n}=\mathbf{5 3 6})$

\begin{tabular}{llcc}
\hline \multicolumn{1}{c}{ Variable } & HR & $\mathbf{9 5} \% \mathbf{C I}$ & $\boldsymbol{P}$ value \\
\hline Resternotomy & 6.7 & $2.04-22.1$ & .002 \\
Preoperative heart failure & 3.6 & $2.04-6.33$ & $<.001$ \\
Previous endocarditis & 2.1 & $0.91-5.05$ & .08 \\
Previous palliation & 2.1 & $1.19-3.77$ & .01 \\
Era of surgery & 1.3 & $0.58-3.07$ & .49 \\
Older age & 1 & $1.01-1.04$ & $<.001$ \\
Etiology of tricuspid regurgitation & 0.9 & $0.25-3.18$ & .86 \\
Gender & 0.7 & $0.37-1.18$ & .1 \\
Tricuspid valve repair & 0.3 & $0.14-0.44$ & $<.001$ \\
\hline
\end{tabular}

$H R$, Hazard ratio; $C I$, confidence interval.

TV dysplasia can be present with PA and an intact ventricular septum. In contrast to the other conotruncal anomalies (transposition of the great arteries, tetralogy of Fallot, truncus), the TV is abnormal from the beginning and might require surgical intervention early in life. In addition, reoperation for recurrent TR is more common in the presence of TV dysplasia and PA with an intact septum. Other diagnoses that can result in TR secondary to a structural abnormality include AVSD and chordal disruption after VSD closure. TR can also be encountered with VSD from other mechanisms: (1) leaflet deformity, (2) TV overriding or straddling, (3) abnormal chordal attachments, (4) Gerbode defect, ${ }^{7}$ and (6) leaflet distortion secondary to VSD patch closure with or without TV leaflet detachment. TV anterior and or septal leaflet detachment is sometimes needed to facilitate VSD closure, which serves to decrease the risk of residual shunt or heart block. The potential disadvantage with this technique is TV leaflet distortion and secondary regurgitation. In the series reported by Bol-Raap and colleagues, ${ }^{8} 188$ patients underwent VSD closure from 1992 to 2001. Temporary detachment of the TV was performed in 46 patients $(24 \%)$. Trivial TR was present in 10 patients $(22 \%)$ postoperatively among the detachment group and in 57 patients from the nondetachment group $(40 \%)$. No patients required reoperation for TV dysfunction during a mean follow-up period of 2.6 years. $^{8}$ Tatebe and colleagues ${ }^{9}$ recommended avoidance of TV detachment in small infants and those with Down syndrome because of the high incidence of TR.

TV regurgitation can also occur from annular dilatation. This can occur with a left-to-right shunt, such as is seen with

TABLE 4. Multivariate hazard ratios for late death for all patients $(\mathbf{n}=\mathbf{5 3 6})$

\begin{tabular}{lccr}
\hline \multicolumn{1}{c}{ Variable } & HR & $\mathbf{9 5} \%$ CI & $\boldsymbol{P}$ value \\
\hline Resternotomy & 12 & $3.24-41.31$ & $<.001$ \\
Preoperative heart failure & 3.3 & $1.82-6.12$ & $<.001$ \\
Previous palliation & 2.2 & $1.2-3.96$ & .009 \\
Older age & 1 & $1.01-1.04$ & .003 \\
Tricuspid repair & 0.4 & $0.19-0.65$ & .001 \\
\hline
\end{tabular}

$H R$, Hazard ratio; $C I$, confidence interval.

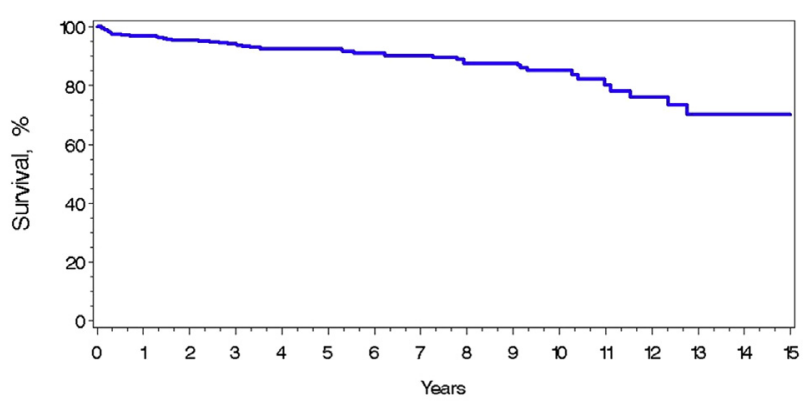

A

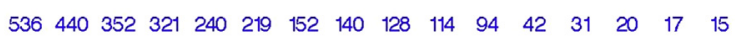

Late Morality by ype (Eanty Moralily were exclubeos)

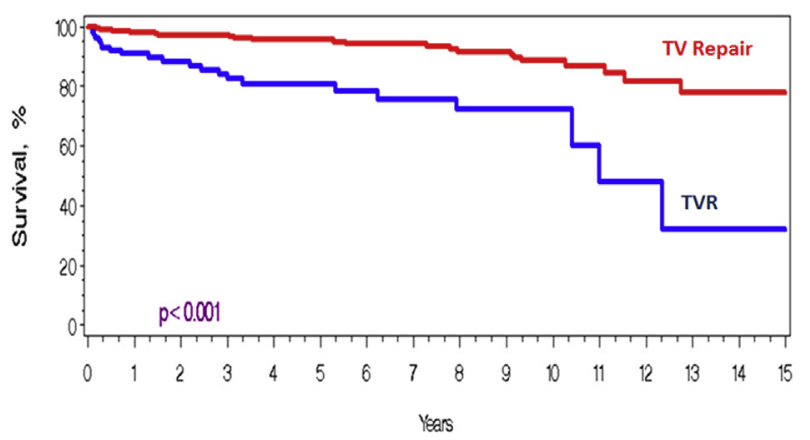

B TV Repair $431360289263195 \quad 181124 \quad 116 \quad 105 \quad 94 \quad 80 \quad 38 \quad 27 \quad 18 \quad 15 \quad 14$ $\begin{array}{lllllllllllllllll}\text { B TVR } & 105 & 80 & 63 & 53 & 45 & 38 & 28 & 24 & 23 & 20 & 14 & 4 & 4 & 2 & 2 & 1\end{array}$

FIGURE 1. Kaplan-Meier graphs showing (A) the overall survival of patients after tricuspid valve surgery, and (B) survival difference between tricuspid valve repair and replacement groups. TVR, Tricuspid valve replacement; $T V$, tricuspid valve.

an atrial septal defect or partial anomalous pulmonary venous connection, from RV dilatation secondary to pulmonary regurgitation, or from increased RV pressure secondary to RV outflow tract obstruction..$^{5}$ Concomitant TV repair at PVR is still controversial, because the long-term effects of PVR on the RV size and secondary TR is unclear. Kogon and colleagues ${ }^{10}$ reported the

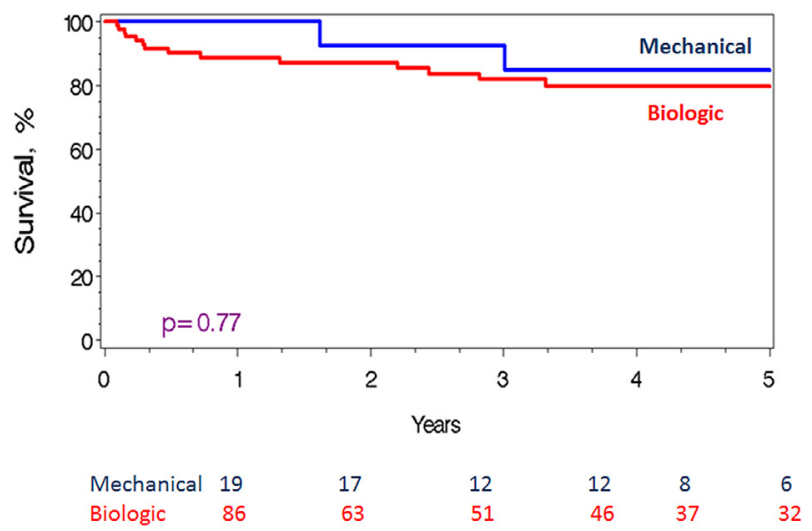

FIGURE 2. Kaplan-Meier graphs showing late survival of patients after tricuspid valve replacement with mechanical versus biologic valves. 


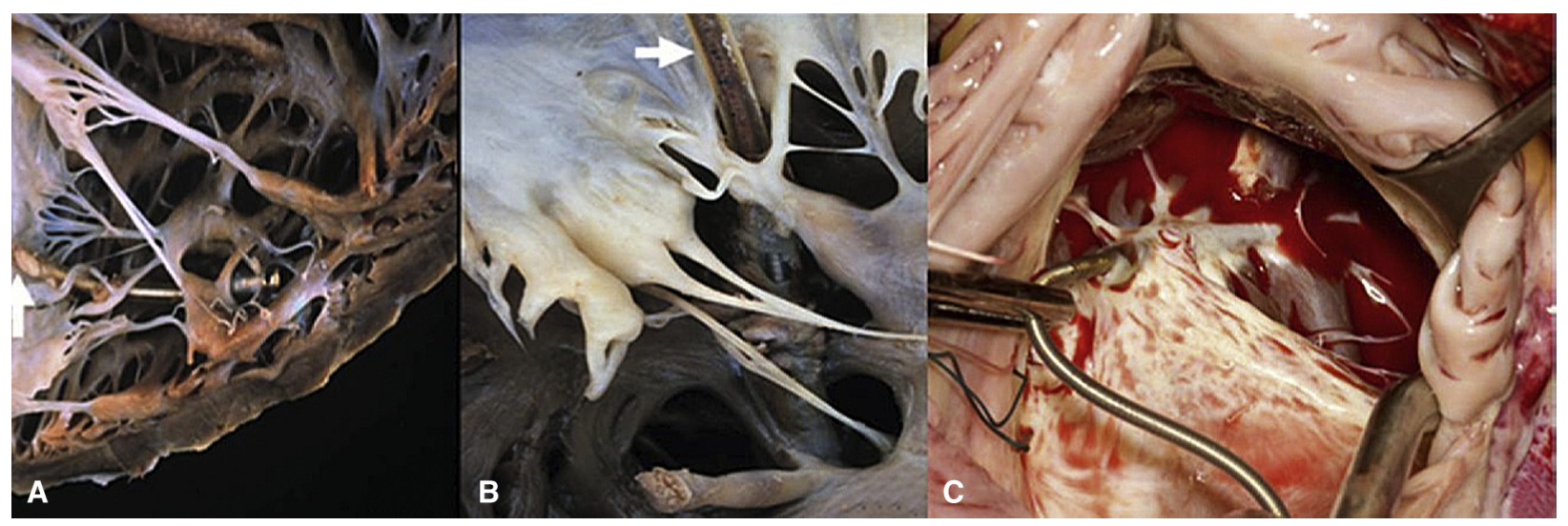

FIGURE 3. A and B, Pathologic specimens and (C) intraoperative photograph showing perforation or entanglement of the tricuspid valve leaflet with pacemaker or cardioverter-defibrillators lead (arrows). Reprinted with permission. ${ }^{1}$

outcomes of 35 patients who underwent PVR subsequent to repair of tetralogy of Fallot or congenital pulmonary stenosis from 2002 to 2008. These patients had had at least moderate TR at PVR; however, the degree of TR and RV dilatation had decreased significantly at 1 month postoperatively, with no additional improvement seen at late follow-up. ${ }^{10}$

Atrial and ventricular arrhythmias are a frequent late complication in congenital heart disease, and the need for a pacemaker or ICD has been increasing. We identified 67 patients $(12 \%)$ who had undergone surgical intervention for "lead-induced" TR. The following mechanisms of TR were identified: (1) leaflet perforation, (2) lead entanglement of the TV apparatus, (3) lead impingement of the TV leaflets, and (4) lead adherence to the TV (Figure 3). TV replacement was required in 38 patients $(56 \%)$, and repair was possible in $29(44 \%)$.

In general, the approach to TV repair in patients with a structural abnormality of the valve often requires attention to the abnormality of the subvalvular apparatus or the leaflet. Specifically, annuloplasty techniques alone are usually inadequate to obtain a durable result when the mechanism is a structural abnormality and not annular dilatation. The recent application of the cone technique and its modifications for $\mathrm{EM}^{2,11}$ can be helpful in optimizing successful TV repair in the presence of structural abnormalities. This can include resection of dysplastic leaflet tissue, approximation of leaflet defects or zones of apposition, placement of artificial Gore-Tex (W. L. Gore) chordae, leaflet augmentation for deficient leaflets, papillary muscle head approximation, leaflet edge incisions to create neochordae, and so on. The addition of an annuloplasty band is important to stabilize the repair that might have included $\geq 1$ of these techniques. Repair techniques in the presence of a permanent pacemaker or ICD lead vary and will depend on the degree of damage to the TV leaflets. However, they can include $\geq 1$ of the following: (1) moving the lead away from the damaged leaflet, (2) suture repair of the leaflet defect, and (3) repositioning the lead by suture fixation at either the inferoseptal or anteroinferior commissure. Ringed annuloplasty is also usually performed (Figure 4).

Raikhelkar and colleagues ${ }^{12}$ reported the outcome of TV repair versus replacement in 56 consecutive adult patients undergoing isolated TV surgery from 1998 to 2010 (9 patients had congenital TR). No difference was found in survival between repair and replacement, but the investigators recommended timely referral to surgery before the onset of heart failure. In the report by Chen and colleagues, ${ }^{13} 77$ patients underwent repeat sternotomy for TV surgery from 2005 to 2010 . TV repair was possible in 44 patients $(57 \%)$. Compared with the repair group, greater morbidity and reduced late survival were found in the replacement group, and these investigators also recommended earlier intervention before the onset of decompensated heart failure. ${ }^{13}$

TV repair versus replacement has not been previously studied in patients with congenital heart disease owing to the heterogeneity of TR in this population. In the present study, valve replacement was more likely and occurred in $35 \%$ of the patient population if there were $\geq 4$ previous sternotomies compared with $20 \%$ if they had undergone $\leq 3$ previous sternotomies. The multivariate analysis in our study demonstrated improved late survival with TV repair compared with TV replacement (Figure 1,B). However, late reoperation for recurrent TR was a significant risk factor for late mortality, emphasizing the importance of obtaining a good to excellent result with TV repair-ideally striving for mild or less regurgitation on the intraoperative transthoracic echocardiogram. Our approach in children is to accept moderate or less regurgitation intraoperatively; however, we counsel the patient and family that reoperation for progressive TR is more likely. In the adult age group, we are less likely to accept moderate or more TR 


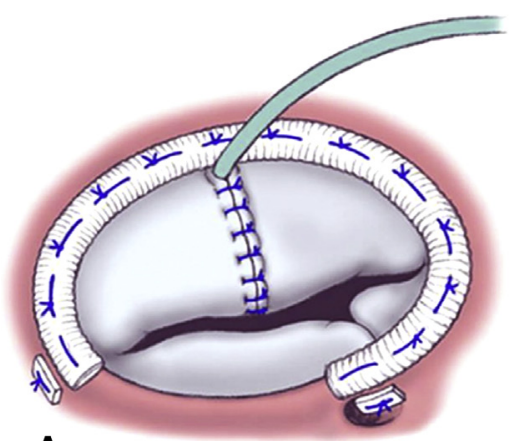

A

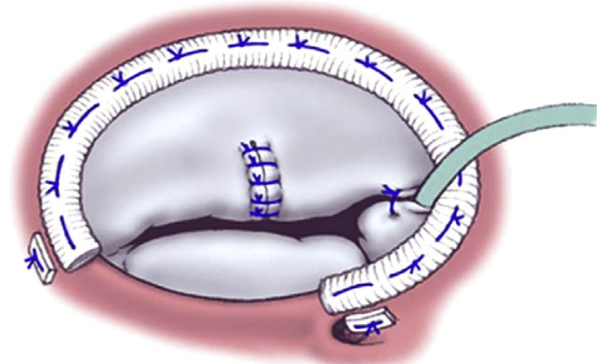

B

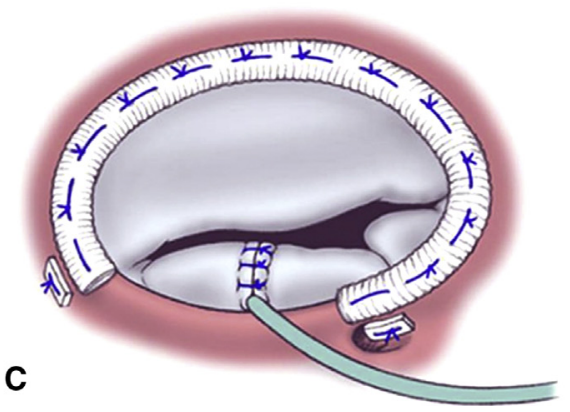

FIGURE 4. A-C, In the presence of pacemaker or cardioverter defibrillator leads, repair techniques vary and depend on the degree of damage of leaflets. In the absence of extensive leaflet damage, valve repair is preferred. It usually involves removing (incising) the lead away from the damaged leaflet, and suture repair of the leaflet defect if present and repositioning the lead by suture fixation against the annulus. Most often suture fixation is in the recess of either the inferoseptal or anteroinferior commissure. Finally, ringed annuloplasty is performed. Reprinted with permission. ${ }^{1}$

intraoperatively and would consider TV replacement, particularly when the patient has already had multiple previous operations, because we have previously shown a significant increase in early and late mortality at sternotomy number 5 and beyond. ${ }^{14,15}$

\section{CONCLUSIONS}

Important TR can occur with a variety of congenital diagnoses. Early mortality has been low and late survival is superior with tricuspid repair compared with TV replacement. Surgical treatment of TR in patients with congenital heart disease should be performed before the onset of heart failure.

\section{References}

1. Said SM, Burkhart HM, Dearani JA. Surgical management of congenital (nonEbstein) tricuspid valve regurgitation. Semin Thorac Cardiovasc Surg Pediatr Card Surg Annu. 2012;15:46-60.

2. Dearani JA, Said SM, O'Leary PW, Burkhart HM, Barnes RD, Cetta F. Anatomic repair of Ebstein's malformation: lessons learned with cone reconstruction. Ann Thorac Surg. 2013;95:220-6.

3. Said SM, Burkhart HM, Schaff HV, Connolly HM, Phillips SD, Suri RM, et al. Late outcome of repair of congenital coronary artery fistulas - a word of caution. J Thorac Cardiovasc Surg. 2013;145:455-60.

4. Nath J, Foster E, Heidenreich PA. Impact of tricuspid regurgitation on long-term survival. J Am Coll Cardiol. 2004;43:405-9

5. Messika-Zeitoun D, Thomson H, Bellamy M, Scott C, Tribouilloy C, Dearani J, et al. Medical and surgical outcome of tricuspid regurgitation caused by flail leaflets. J Thorac Cardiovasc Surg. 2004;128:296-302.

6. Becker AE, Becker MJ, Edwards JE. Pathologic spectrum of dysplasia of the tricuspid valve: features in common with Ebstein's malformation. Arch Pathol. 1971;91:167-78.
7. Wasserman SM, Fann JI, Atwood JE, Burdon TA, Fadel BM. Acquired left ventricular right atrial communication: Gerbode-type defect. Echocardiography. 2002; 19:67-72.

8. Bol-Raap G, Weerheim J, Kappetein AP, Witsenburg M, Bogers AJ. Follow-up after surgical closure of congenital ventricular septal defect. Eur J Cardiothorac Surg. 2003;24:511-5.

9. Tatebe S, Miyamura H, Watanabe H, Sugawara M, Eguchi S. Closure of isolated ventricular septal defect with detachment of the tricuspid valve. J Card Surg. 1995; 10:564-8.

10. Kogon B, Patel M, Leong T, McConnell M, Book W. Management of moderate functional tricuspid valve regurgitation at the time of pulmonary valve replacement: is concomitant tricuspid valve repair necessary? Pediatr Cardiol. 2010; 31:843-8.

11. Dearani JA, Said SM, Burkhart HM, Pike RB, O’Leary PW, Cetta F. Strategies for tricuspid re-repair in Ebstein malformation using the cone technique. Ann Thorac Surg. 2013;96:202-10.

12. Raikhelkar J, Lin HM, Neckman D, Afonso A, Scurlock C. Isolated tricuspid valve surgery: predictors of adverse outcome and survival. Heart Lung Circ. 2013;22:211-20

13. Chen SW, Tsai FC, Tsai FC, Chao YK, Huang YK, Chu JJ, et al. Surgical risk and outcome of repair versus replacement for late tricuspid regurgitation in redo operation. Ann Thorac Surg. 2012;93:770-5

14. Holst KA, Dearani JA, Burkhart HM, Connolly HM, Warnes CA, Li Z, et al Reoperative multivalve surgery in adult congenital heart disease. Ann Thorac Surg. 2013;95:1383-9.

15. Holst KA, Dearani JA, Burkhart HM, Connolly HM, Warnes CA, Li Z, et al. Risk factors and early outcomes of multiple reoperations in adults with congenital heart disease. Ann Thorac Surg. 2011;92:122-8.

\section{Discussion}

Dr Charles D. Fraser (Houston, Tex). I have no disclosures.

I congratulate Dr Said. I have heard him present before, and this is another example of a beautifully presented paper and elegant slides and very clear, and I thank you for that. 
You and your colleagues from the Mayo Clinic have conducted yet another elegant study and presentation, and all of us who practice congenital heart surgery owe a debt of gratitude to the Mayo surgical legacy of innovation and scholarly assessment of results that was emphasized so nicely by our current president, Dr Schaff, in his presentation earlier today.

This very large retrospective review of 553 patients with congenital heart disease and associated TV corrective surgery provides a window of opportunity for our understanding of the results after TV operations in an admittedly very heterogeneous population of patients, as you noted in your presentation and report.

The central question of focus in all of our minds I expect is "What are we to do with this information in modifying our approach to the TV in patients with congenital heart disease?" As such, I ask the following questions, and I will ask them in series, and you can just answer them before going to the next.

The first, in your analysis of the predictors of mortality, you found TV replacement, repeat sternotomy, previous palliation, and older age to be risk factors. Given that, with the exception of valve replacement, this accounts for almost everyone who might ultimately need TV surgery after previous congenital heart surgery. How are we to use these data in decision-making about who needs TV surgery?

Dr Said. Thank you, Dr Fraser, for your comments.

First, regarding the predictors of mortality. The message from this report, despite this heterogeneous group, is we should operate earlier when significant TR is present and not consider it as a benign lesion. Heart failure and resternotomy are also predictors of mortality. Valve repair is almost always preferred, but when we cannot achieve a successful repair of moderate or less regurgitation, it is reasonable to consider TV replacement, because it treats right-sided heart failure and might delay the next sternotomy compared with a poor tricuspid valve repair.

Dr Fraser. Sort of as an extension of that question, and I had the opportunity to review the report, and I realize you cannot share all that information in a brief presentation such as this, but in the report, you noted that your indication for surgery on a TV was moderate or more TR. However, you also noted that you and others have clearly shown that, for example, in a patient with late repaired tetralogy of Fallot who had severe pulmonary valve insufficiency and a dilating right ventricle, if you placed a competent pulmonary valve, the TV would get better.

Thus, from this study and your own experience, how did these data change your thinking and what are your current indications for intervention on the TV?

Dr Said. In general, the current indication for TV intervention is the presence of severe TR and symptoms, which can include rightsided heart failure, decreased exercise tolerance, and/or fatigue. When TR is secondary, for example, in the setting of pulmonary regurgitation, we intervene on the TV when grade III or IV TR is present, a structural abnormality is present with the valve (eg, chordal rupture or leaflet defect), when severe annular dilatation is present, or when the pulmonary artery pressures are elevated. In patients who are apparently asymptomatic, we perform exercise testing. If the test results are abnormal, we would lean toward TV repair. When the exercise testing results are normal, we monitor the RV size with serial imaging, magnetic resonance imaging most commonly. In general, the overall message is earlier intervention for TR.

Dr Fraser. Just to follow up on the indications a bit. Would you suggest the addition of more objective measures such as stress testing or magnetic resonance imaging evaluation of the right ventricle as additional adjuncts to the decision tree?

Dr Said. Yes, we believe magnetic resonance imaging is the most valuable imaging study, because it provides an accurate assessment of RV size and function. We find echocardiography to be most helpful for analysis of the valve anatomy.

Dr Fraser. Finally, is the title a bit misleading? Can we, from these data in a retrospective analysis, really conclude that it is the operation that is the difference or is it the patients who undergo the operation? In other words, is it justified to state that the TV repair is protective over TV replacement or is it the patients who require the replacement who are at higher risk, not the operation?

Dr Said. This is another important point. The clinical status of the patients undergoing surgery, particularly those with right-sided heart failure, also affects the outcome of reoperation. The published data and our study have demonstrated that when surgery is performed in the setting of class IV heart failure, early mortality is increased and late survival is reduced. The challenge with this study is that we had a heterogeneous group of lesions, making it more difficult to differentiate the outcomes on the basis of repair versus replacement alone.

Dr Fraser. Again, thank you for a very nice presentation.

Dr Christopher A. Caldarone (Toronto, Ontario, Canada). May I extend that question just a little bit. You identify congestive heart failure as 1 of the most important risk factors for death. It is a modifiable risk factor. Thus, how do you use this information from 2 directions: (1) when a patient presents with congestive heart failure, do you modify the preoperative management in accordance with this finding before you bring them to the operating room; and (2) what patients have too much congestive heart failure to undergo surgery? Are there any patients you would not operate on and what parameters would you use to exclude a patient from surgery?

Dr Said. Modifying heart failure before surgery will depend on the urgency of the situation. Most patients can be medically optimized as an outpatient. Some patients might be admitted preoperatively for medical refinement, including aggressive diuresis and fluid restriction and, rarely, intravenous inotropic support.

Dr Caldarone. Thus, it might be possible through an analysis of your database to identify when you actually mitigated the effect of congestive heart failure by those patients who were treated preoperatively in the aggressive fashion you described?

Dr Said. Yes.

Dr Caldarone. What about patients you had excluded from surgery, does that ever happen?

Dr Said. Patients who might not be suitable for surgery include those with significant left ventricular dysfunction, severe RV dysfunction, and those with liver dysfunction. In general, if the synthetic function of the liver is intact, we offer surgery. Young patients with severe biventricular dysfunction or multiple organ system dysfunction usually undergo evaluation for potential multiple organ transplantation.

Dr Christopher Baird (Boston, Mass). With regard to the variables of reoperative TV repair in resternotomy, it seems that 
you have identified at least resternotomy as a risk factor. With your large database, were you able to identify how this might guide your ability to decide whether to repair the valve again or replace the valve?

Dr Said. Some situations might allow valve repeat repair. In general, if the leaflet tissue and subvalvular apparatus are pliable and thin, we attempt repeat repair. The various techniques for repair of the Ebstein TV can be applied and can help facilitate valve repair. As the tricuspid leaflet tissue becomes thicker and more rigid, repeat repair becomes more difficult and replacement more likely. We have previously published information related to the sternotomy number and operative risk. The operative risk increases at the fourth and fifth sternotomy. Consequently, the sternotomy number also affects the decision to replace versus repeat repair. We are more likely to replace the TV at the fourth or fifth redo unless it is a straightforward TV repair with a high probability of good valve competence.

Dr Pedro J. del Nido (Boston, Mass). Can I follow up on the issues of functional TR. Can you give us a bit more information on the patient groups, how many of these patients had left-sided disease at the same time you were treating the TV and was that taken into consideration as 1 of the risk factors?

Dr Said. In general, the left ventricular function was normal for most patients. Approximately $30 \%$ of patients underwent a left-sided procedure; mitral valve surgery was most common. Importantly, however, this study focused on patients whose main problem was TR. Specifically, this was their main hemodynamic lesion and the main indication for surgery. Left-sided lesions, when present, were usually of moderate severity (eg, moderate mitral regurgitation) and were addressed during surgery but were not the main indications for surgery.

Dr del Nido. Because 1 of the points that is well documented now in adult published studies is functional TR in the face of mitral disease and that mortality is significantly greater if you do not address it at the same time as the mitral valve. I am wondering whether you included the functional TR patients into a congestive heart failure category. How many of those patients actually had also either left atrioventricular valve or some sort of left-sided heart disease as a part of their congestive heart failure?

Dr Said. I think that would be about $25 \%$ or $30 \%$ of these patients also had left-sided problems. A mitral valve procedure was performed in $23 \%$ and an aortic valve procedure in $9 \%$. In general, these left-sided lesions were not severe but were abnormal enough that an intervention was performed because surgery was advised for right-sided heart failure. It is reasonable to assume that patients with severe left-sided abnormalities that are corrected (eg, severe aortic stenosis or severe mitral regurgitation) will result in some improvement in functional TR.

Dr del Nido. Thus, I guess the next question would be "If you have a patient who you are operating on for the left-sided atrioventricular valve, let us say a canal, who has moderate TR, they have symptoms, that is why you are operating on the left side, what would be your decision tree as far as whether to intervene on the right side as well?

Dr Said. If severe TR is present or the TV has any structural abnormalities on preoperative imaging or intraoperative transesophageal echocardiography, we repair the TV. If the TR is moderate and no tricuspid structural abnormality is present and a severe left-sided lesion is also present that is being fixed, we usually leave the TV alone.

Dr del Nido. Although many times the problem is not so much annular, I mean there are subvalvular problems in these patients, some of the subvalvular apparatus is quite abnormal. In your images, or at least in the schematics you showed, it seems as if most of the work that you are focusing on is annuloplasty and some commissuroplasty. Is there any work that is also done in the subvalvular apparatus?

Dr Said. Repair maneuvers are directed at the abnormality. In most situations in this patient cohort, this was at the leaflet or annular level. The subvalvular apparatus was addressed when a problem was identified in this area-unsupported segments might be treated with artificial chordae, mobilization of papillary muscles, approximation of $\geq 1$ of the papillary muscles to the ventricular septum, or creation of autologous chordae for the tethered leading edge are some examples of subvalvular maneuvers. 